\title{
Design and Preparation of Single-Chain Nanocarriers Mimicking Disordered Proteins for Combined Delivery of Dermal Bioactive Cargos ${ }^{\mathbf{a}}$
}

Ana Sanchez-Sanchez, Somayeh Akbari, Angel J. Moreno, Federica Lo Verso, Arantxa Arbe, Juan Colmenero, José A. Pomposo*

\begin{abstract}
A. Sanchez-Sanchez
Centro de Física de Materiales (CSIC, UPV/EHU) - Materials Physics Center, Paseo Manuel de Lardizábal 5, 20018 San Sebastián, Spain, Departamento de Física de Materiales, Universidad del País Vasco (UPV/EHU), Apartado 1072, 20800 San Sebastián, Spain

Angel J. Moreno, Federica Lo Verso, Arantxa Arbe

Centro de Física de Materiales (CSIC, UPV/EHU) - Materials Physics Center, Paseo Manuel de Lardizábal 5, 20018 San Sebastián, Spain
\end{abstract}

\section{S. Akbari}

Laboratory for Tribology and Surface Nanotechnology, Bogišičeva 8, 1000 Ljubljana, Slovenia

\section{J. Colmenero}

Centro de Física de Materiales (CSIC, UPV/EHU) - Materials Physics Center, Paseo Manuel de Lardizabal 5, 20018 San Sebastián, Spain, Departamento de Física de Materiales, Universidad del País Vasco (UPV/EHU), Apartado 1072, 20800 San Sebastián, Spain, and Donostia International Physics Center (DIPC), Paseo Manuel de Lardizabal 4, 20018 San Sebastián, Spain

\section{J. A. Pomposo}

Centro de Física de Materiales (CSIC, UPV/EHU) - Materials Physics Center, Paseo Manuel de Lardizabal 5, 20018 San Sebastián, Spain, Departamento de Física de Materiales, Universidad del País Vasco (UPV/EHU), Apartado 1072, 20800 San Sebastián, Spain, and IKERBASQUE - Basque Foundation for Science, Alameda Urquijo 36, 48011 Bilbao, Spain E-mail: josetxo.pomposo@ehu.es

Inspired by the multi-functionality of vitamin D-binding protein and the multiple transientbinding behavior of some intrinsically disordered proteins (IDPs), we have designed, prepared and characterized a polymeric platform for combined delivery of dermal protective and anticancer bioactive cargos based on artificial single-chain nano-objects mimicking IDPs. For the first time ever, simultaneous delivery of folic acid, or vitamin $\mathrm{B}_{9}$, and hinokitiol, a 
relevant natural bioactive compound that exhibits anticancer activity against human malignant melanoma cells, from these multi-directionally self-assembled unimolecular nanocarriers is illustrated.

\section{Introduction}

The vitamin D-binding protein (DBP) or GC-globulin, identified about a half a century ago, ${ }^{[1]}$ is a multi-functional protein found in human serum that binds and transports various forms of vitamin D, as well as other biomolecules like actin or fatty acids. DBP's main function is to retain vitamin $\mathrm{D}$ for the organism and make it available to various tissues and cell types for usage. ${ }^{[2]}$ Various forms of vitamin D, such as D3 (cholecalciferol), D2 (ergocalciferol), 25D (25-hydroxy-vitamin D) and 1,25D (1,25-dihydroxy-vitamin D), the active form of vitamin D, are bound to DBP via a surface vitamin D-binding cleft in one of the three domains of the protein. ${ }^{[3]}$ The affinity constants for 25D-, 1,25D-, actin- and fatty acid-binding to BDP are $7 \times 10^{8} \mathrm{M}^{-1}, 4 \times 10^{7} \mathrm{M}^{-1}, 1 \times 10^{9} \mathrm{M}^{-1}$ and $6-7 \times 10^{5} \mathrm{M}^{-1}$, respectively. ${ }^{[4]}$

In contrast to globular proteins adhering to the classical structure-function paradigm, intrinsically disordered proteins (IDPs) lack a well-defined unique structure, adopting in solution a complete ensemble of rapidly interconverting conformations. ${ }^{[5]}$ Interestingly, in spite of its disordered structure, IDPs can bind several ligands, such as ions, small organic molecules, other proteins and nucleic acids (RNA, DNA). The interplay between structure and function in IDPs has not been recognized until very recently and it is not completely understood yet. $^{[6]}$ Although some IDPs are simple coils that bond together other proteins, most of them have transient-binding or permanent-binding recognition sites. Moreover, a large number of IDPs exhibit what is referred to as binding promiscuity. ${ }^{[7]}$

Recently, we have reported that the structure of multi-directionally self-assembled singlechain polymer particles (SCNPs) under good solvent conditions closely resemble that of IDPs 
in solution. ${ }^{[8]}$ Kratky plots from small angle neutron scattering (SANS) measurements showed a structure factor in close analogy to that of disordered multi-domain proteins (e.g., p67 $7^{\text {phox }}$ ) so, from a structural point of view, they could be considered, to a good approximation, as artificial mimics of IDPs and denoted as IDPMs. It is worth mentioning that the Kratky plot has the capacity to enhance particular features of scattering profiles, thus allowing easier identification of degrees of compactness. ${ }^{[5]}$ Hence, according to SANS results SCNPs in good solvents show multiple local-scale domains separated by flexible linkers, whereas SCNPs in the dry state adopt a globular morphology, as observed by TEM. Moreover, in this previous study the ability of the SCNPs to deliver vitamin $\mathrm{B}_{9}$ in water at neutral $\mathrm{pH}$ was assessed. The delivery curve of vitamin $\mathrm{B}_{9}$ was fitted to a power law model from which the value of the release exponent was found to be $c a$. 0.5 , suggesting that the process proceeds through a Fickian diffusion mechanism and completes in less than 5 hours. ${ }^{[8]}$

We hypothesized that in analogy to some disordered proteins, IDPMs could also have some degree of binding promiscuity towards some relevant bioactive compounds that could be then delivered to water in a $\mathrm{pH}$-dependent manner. In particular, we investigate herein the potential use of SCNPs as artificial IDPM nanocarriers for simultaneous delivery of both dermal protective and anticancer cargos (see Fig. 1A). As a model system of dermal protective compounds, we selected folic acid (also known as folate or vitamin $\mathrm{B}_{9}$ ), $\mathbf{1}$. According to several in vitro and in vivo studies, dermal delivery of $\mathbf{1}$ using topical formulations offers a treatment option for photo-aged skin. ${ }^{[9,10]}$ As a natural anticancer drug, we selected hinokitiol ( $\beta$-thujaplicin), 2, as a tropolone-related compound found in heartwood of cupressaceous plants that exhibits anticancer activity against human malignant melanoma cells $^{[11]}$ as well as other cancer cell lines, including the human leukemic cell K562 and the human stomach cancer cell KATO-III. ${ }^{[12,13]}$ The selective anticancer mechanism of $\mathbf{2}$ in melanoma cancer cells versus normal human melanocytes has been recently reported to involve strong G1 cell cycle arrest due to p27 protein accumulation, down-regulation of Skp2 
proteins, and inhibition of Cdk2 kinase activity. ${ }^{[14]}$ As a proof of concept we will demonstrate, for the first time ever, the simultaneous delivery of $\mathbf{1}$ and $\mathbf{2}$ to water solutions at different $\mathrm{pH}$ values from SCNPs as artificial IDPM nanocarriers.

\section{Results and Discussion}

\subsection{Design of intrinsically disordered protein mimetics (IDPMs)}

The design of intrinsically disordered protein mimetics, IDPMs, was guided by molecular dynamics (MD) simulations. In particular, coarse-grained MD simulations were performed to investigate the effect of different parameters, such as precursor chain length and functionality of reactive groups along the chain on the final structure and morphology of the resulting IDPMs in solution. A detailed description of the MD simulations performed is provided in the Supplementary Information (SI). In brief, a precursor chain of $\mathrm{N}$ beads of radius $\sigma$ having a fraction of monomers $\mathrm{x}=0.2$ of functionality $\mathrm{f}$, able to form permanent intrachain cross-links, was first equilibrated as a self-avoiding random walk (i.e. good solvent conditions). Then, MD simulations were carried out allowing the progressive formation of irreversible links between reactive monomers in the chain if the distance between monomers is lower than a certain capture radius $(1.3 \sigma)$. Once the intrachain cross-linking process was completed, the average radius of gyration $\left(\mathrm{R}_{\mathrm{g}}\right)$ of the resulting nano-objects was determined. ${ }^{[15-21]}$

Based on the MD simulation results, IDPM size was found to depend more strongly on precursor length than on the functionality of the reactive groups. In order to define the structure of the nanoparticles taking into account multi-body correlations among the monomers, we use the Euler characteristic as a topological fingerprint ${ }^{[2,23]}$. In Fig. 2 we can observe a deep minimum $(\mathrm{R} \approx 0.5 \sigma)$ which give us informations on the typical (limited) hole size. The deep minimum and the lack of any further positive peak suggest an elongated sparse structure instead of a compact, globular structure. No significant differences in the typical 
morphology of the resulting IDPMs were observed by increasing the functionality of the reactive groups in the polymer precursor. On the other hand, a broad range of morphologies were observed as a consequence of the statistical placement of reactive groups along the individual chains and the stochastic nature of the cross-linking process (see Fig. S1). Both the precursor chains and the nano-objects follow a $\mathrm{R}_{\mathrm{g}}{ }^{2} \propto \mathrm{N}^{2 v}$ scaling law; the values of the $v$ exponent were found to be $v=0.63 \pm 0.01, v=0.57 \pm 0.01$ and $v=0.55 \pm 0.01$ for the precursor, bifunctional and trifunctional cross-linked nanoparticles, respectively (see Fig. S2). Hence, the reduction in size upon IDPM formation from the linear precursor was found to grow with increasing precursor size. Based on the obtained scaling laws, precursor chains $(\mathrm{f}=$

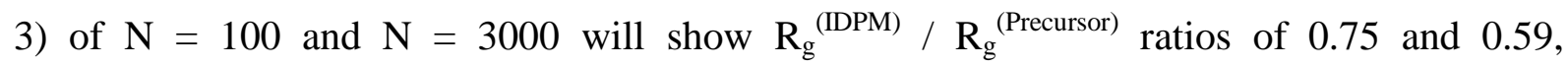
respectively.

\subsection{Preparation and characterization of IDPMs}

Guided by the MD simulation results, we target a precursor molecular weight of $3 \times 10^{5} \mathrm{Da}$ having a representative amount of cross-linking groups of $25 \mathrm{~mol} \%$ for which we expect a $\mathrm{R}_{\mathrm{g}}{ }^{\text {(IDPM) }} / \mathrm{R}_{\mathrm{g}}{ }^{\text {(Precursor) }}$ ratio of 0.59 when collapsed to individual IDPM under good solvent conditions. Such a high molecular weight will allow us to determine the evolution of $\mathrm{R}_{\mathrm{g}}$ during IDPM formation via SEC/SLS measurements. In this sense, SEC chromatography discriminates macromolecules according to their hydrodynamic radius $\left(\mathrm{R}_{\mathrm{h}}\right)$ which is roughly inversely proportional to SEC elution time $\left(t_{R}\right)$, whereas the SLS technique provides values of both absolute molecular weight $\left(\mathrm{M}_{\mathrm{w}}\right)$ and $\mathrm{R}_{\mathrm{g}}$. The polymer precursor was synthesized from commercially available methyl methacrylate (MMA) and (2-acetoacetoxy)ethyl methacrylate (AEMA) monomers via RAFT copolymerization (see Fig. 1B), showing an average $\mathrm{R}_{\mathrm{g}}=14.8$ $\pm 0.5 \mathrm{~nm}$, relatively narrow polydispersity $\left(\mathrm{M}_{\mathrm{w}} / \mathrm{M}_{\mathrm{n}}=1.37\right)$ and a random distribution of reactive $\beta$-ketoester moieties from AEMA repeat units, as determined by ${ }^{1} \mathrm{H}$ NMR spectroscopy. To construct the IDPMs, the polymer precursor was individually collapsed 
under controlled stoichiometric and dilution conditions in tetrahydrofuran (THF) at r.t. through multidirectional Michael addition reactions involving the single-chain $\beta$-ketoester groups, as Michael donors, and external acrylate-based cross-linkers of functionality $f=3$, as Michael acceptors. ${ }^{[8]}$ This method produces robust, covalent bonded single-chain nanoobjects. $^{[24,25]}$ The effect of precursor concentration during the synthesis of IDPMs on nanoobject size and size distribution is summarized in Table $\mathbf{1}$, as determined by combined SEC/SLS and complementary DLS measurements. By working at $3 \mathrm{mg} / \mathrm{ml}$, an increase in the value of the actual molecular weight was observed even when a moderate reduction in the values of $R_{h}$ and $R_{g}$ was clearly observed. Upon decreasing the precursor concentration to 1 $\mathrm{mg} / \mathrm{ml}$, the absolute molecular weight values at the SEC peak maximum of the precursor and the IDPMs were found to be very similar, and a larger reduction in the values of $R_{h}$ and $R_{g}$ was found. No further reduction was observed by working at precursor concentrations $<1$ $\mathrm{mg} / \mathrm{ml}$. In this sense, it is worth mentioning that potential intermolecular cross-linking events are increasingly avoided by passing from a semi-concentrated to a highly diluted solution. ${ }^{[26,27]}$ A vertical shift (downward) in the curve of $R_{g}$ versus $t_{R}$ was observed upon nano-object formation (see Fig. 3A) which can be attributed to a change in the $R_{g} / R_{h}$ ratio on passing from linear coils to IDPMs. For a flexible polymer chain under good solvent conditions $\mathrm{R}_{\mathrm{g}} / \mathrm{R}_{\mathrm{h}} \approx 1.56$, whereas for globular particles $\mathrm{R}_{\mathrm{g}} / \mathrm{R}_{\mathrm{h}} \approx 0.77 .{ }^{[28]}$ IDPMs synthesized at $3 \mathrm{mg} / \mathrm{ml}$ show a ratio of $\mathrm{R}_{\mathrm{g}} / \mathrm{R}_{\mathrm{h}}=1.25$, whereas IDPMs synthesized at $1 \mathrm{mg} / \mathrm{ml}$ display $\mathrm{R}_{\mathrm{g}} / \mathrm{R}_{\mathrm{h}}$ $=1.05$, the latter being a value intermediate between those expected for a coil and a globule. The collapse degree $\left(\mathrm{R}_{\mathrm{g}}{ }^{(\mathrm{IDPM})} / \mathrm{R}_{\mathrm{g}}{ }^{\text {(Precursor) }}\right)$ for nanoparticles $(\mathrm{f}=3)$ synthesized at $1 \mathrm{mg} / \mathrm{ml}$ was 0.55 , in good agreement with the value estimated from MD simulations. According to data summarized in Table 1, IDPM-1 shows a structure more collapsed than IDPM-3.

The typical morphology of the IDPMs under good solvent conditions was deduced from SAXS measurements, showing a Kratky plot resembling that observed in natural intrinsically disordered proteins and very different from that of the linear precursor (see Fig. 
3B). ${ }^{[5]}$ As expected, SAXS results were in good agreement with previous SANS and MD simulation data. ${ }^{[8]}$ Conversely, the morphology of individual IDPMs in the dry state was undoubtedly globular, as observed by TEM, showing collapsed nanoparticles with a spherical shape (Fig. 3C).

\subsection{Combined delivery of dermal bioactive cargos from IDPMs}

To test our hypothesis of IDPM multi-binding ability, the nanocarriers were loaded with both folic acid (1) and hinokitiol (2) and the controlled delivery of these protective and anticancer dermal bioactive cargos, respectively, in water at two relevant $\mathrm{pH}$ values $(\mathrm{pH}=6$ and $\mathrm{pH}=8)$ was monitorized by UV spectroscopy. For quantifying the delivery process, the intensities of the UV absorption maxima of $\mathbf{1}$ and 2 at $283 \mathrm{~nm}$ and $240 \mathrm{~nm}$, respectively, as well as appropriate calibration curves, were employed (see Figs. S3, S4). Figure 3D illustrates the experimental data corresponding to the simultaneous delivery of $\mathbf{1}$ and $\mathbf{2}$ at $\mathrm{pH}=6$ and $\mathrm{pH}=8$ from IDPMs loaded with $51 \%$ of dermal bioactive compounds (ratio $2: \mathbf{1}=0.53$ ). According to these data, on average, there are 410 molecules of $\mathbf{2}$ and 170 molecules of $\mathbf{1}$ per IDPM carrier. The delivery process for both compounds was faster at $\mathrm{pH}=8$ than at $\mathrm{pH}=6$, especially at short delivery time, whereas total delivery was found to take place in about 4 hours at both $\mathrm{pH}$ values. All our attempts to characterize the presumably weak 1/IDPM and 2/IDPM interactions under the high diluted experimental conditions by ${ }^{1} \mathrm{H}$ NMR, UV-vis and FTIR spectroscopies failed. Nevertheless, the simultaneous delivery of $\mathbf{1}$ and $\mathbf{2}$ to water solutions at relevant $\mathrm{pH}$ values from SCNPs as artificial IDPM nanocarriers pave the way to the long-term development of multifunctional topical formulations containing IDPMs.

\section{Conclusion}

Guided by MD simulations results we have developed and characterized single-chain nanocarriers mimicking disordered proteins (IDPMs) for combined delivery of dermal 
bioactive cargos. Hence, MD simulations results have shown that IDPM size depends more strongly on precursor length than on the functionality of the reactive groups in the precursor. By using the Euler characteristic as a topological fingerprint, we have found that the average hole size is similar to the bead size employed in the simulations. Snapshots of the IDPMs showed elongated sparse estructures instead of compact, globular morphologies displaying $\mathrm{R}_{\mathrm{g}}{ }^{(\mathrm{IDPM})} / \mathrm{R}_{\mathrm{g}}{ }^{\text {(Precursor) }}$ ratios between 0.75 and 0.59 depending on the precursor length. Good agreement was found between the collapse degree of IDPMs synthesized via Michael addition-induced self-assembly $\left(\mathrm{R}_{\mathrm{g}}{ }^{(\mathrm{IDPM})} / \mathrm{R}_{\mathrm{g}}{ }^{(\text {Precursor })}=0.55\right)$ and the value estimated from MD simulations $\left(\mathrm{R}_{\mathrm{g}}{ }^{(\mathrm{IDPM})} / \mathrm{R}_{\mathrm{g}}{ }^{\text {(Precursor) }}=0.59\right)$ when intrachain cross-linking was carried out at high dilution. SAXS measurements supported the MD simulation results showing an IDPM form factor in the Kratky plot similar to that observed in natural disordered proteins. TEM pictures, however, displayed a globular IDPM morphology upon drying, pointing to a transition from extended to compact nano-objects when solvent was removed. The multibinding ability of the single-chain nano-objects was demonstrated by simultaneous delivery of hinokitiol and folic acid to water solutions at relevant $\mathrm{pH}$ values paving the way to the development of multifunctional topical formulations containing IDPMs.

Acknowledgements: Financial support from the projects MAT2012-31088 (MINECO) and IT654-13 (GV) is acknowledged. A. S.-S. thanks the PhD grant support of Basque Government.

Received:

Revised:

Published online:

DOI: $10.1002 /$ marc.201300562

Keywords: Nanocarriers; self-assembly; nanoparticles; nanomedicine; drug delivery. 
[1] J. Hirschfeld, Phatol. Microbio. Scand., 1959, 47, 160.

[2] R. Bouillon, “The Vitamin D Binding Protein DBP”, In Vitamin D (3 ${ }^{\text {rd }}$ edn.), Vol. 1; Academic Press, London, 2011.

[3] R. A. Laskowski, N. M. Luscombe, M. B. Swindells, J. M. Thornton, Protein Sci., 1996, $5,2438$.

[4] R. F. Chun, Cell Biochem. Funct., 2012, 30, 445.

[5] P. Bernado, D. I. Svergun, Mol. BioSyst., 2012, 8, 2955.

[6] P. Tompa, K.-H. Han, Phys. Today, 2012, 65, 64.

[7] V. N. Uversky, BBA-Proteins. Proteom., 2013, 1834, 932.

[8] A. Sanchez-Sanchez, S. Akbari, A. Etxeberria, A. Arbe, U. Gasser, A. J. Moreno, J. Colmenero, J. A. Pomposo, ACS Macro Lett., 2013, 2, 491.

[9] A.Knott, H. Mielke, U. Koop, R. Wolber, T. Burkhardt, J.-P.Vietzke, F. Stäb, H. Wenck, S. Gallinat, J. Invest. Dermatol., 2007, 127, 2463.

[10] F. Fischer, V. Achterberg, A. März, S. Puschmann, C.-D. Rahn, V. Lutz, A. Krüger, H. Schwengler, S. Jaspers, U. Koop, T. Blatt, H. Wenck, S. Gallinat, J. Cosmet. Dermatol. 2011, $10,15$.

[11] T. Baba, H. Nakano, K. Tamai, D. Sawamura, K. Hanada, I. Hashimoto, Y. Arima, J. Invest. Dermatol., 1998, 110, 24.

[12] Y. Inamori, H. Tsujibo, H. Ohishi, F. Ishii, M. Mizugaki, H. Aso, N. Ishida, Biol. Pharm. Bull., 1993, 16, 521.

[13] D. Miyamoto, N. Endo, N. Oku, Y. Arima, T. Suzuki, Y. Suzuki, Biol. Pharm. Bull., 1998, $21,1258$.

[14] S. Liu, H. Yamauchi, Cancer Lett. 2009, 286, 240.

[15] K. Kremer, G. S. Grest, J. Chem. Phys., 1990, 92, 5057.

[16] M. P. Allen, D. J. Tildesley, Computer simulation of liquids, Oxford University Press, Oxford (UK), 1989. 
[17] J. A. Izaguirre, D. P. Catarello, J. M. Wozniak, R. D. Skeel, J. Chem. Phys., 2001, 114, 2090.

[18] D. R. Rottach, J. G. Curro, J. Budzien, G. S. Grest, C. Svaneborg, R. Everaers, Macromolecules, 2006, 39, 5521.

[19] B. Capone, I. Coluzza, F. Lo Verso, C. N. Likos, R. Blaak, Phys. Rev. Lett., 2012, 109, 238301.

[20] F. Lo Verso, A. Z. Panagiotopoulos, C. N. Likos, Phys. Rev. E, 2009, 79, 010401.

[21] C. Koch, C. N. Likos, A. Z. Panagiotopoulos, F. Lo Verso, Mol. Phys., 2011, 109, 3049.

[22] C. N. Likos, K. R. Mecke, and H. Wagner, J. Chem. Phys., 1995, 102, 9350.

[23] N. Hoffmann, F. Ebert, C. N. Likos, H. Lowen, and G. Maret, Phys. Rev. Lett., 2006, 97, 078301.

[24] A. Sanchez-Sanchez, I. Pérez-Baena, José A. Pomposo, Molecules, 2013, 18, 3339.

[25] O. Altintas, C. Barner-Kowollik, Macromol. Rapid Commun., 2012, 33, 958.

[26] a) E. Harth, B.V. Horn, V.Y. Lee, D.S. Germack, C.P. Gonzales, R.D. Miller, C. J. Hawker, J. Am. Chem. Soc., 2002, 124, 8653; b) B. T. Tuten, D. Chao, C. K. Lyon, E. B. Berda, Polym. Chem., 2012, 3, 3068; c) I. Perez-Baena, F. Barroso-Bujans, U. Gasser, A. Arbe, A. J. Moreno, J. Colmenero, J. A. Pomposo, ACS Macro Lett., 2013, 2, 775.

[27] a) J. A. Pomposo, I. Perez-Baena, L. Buruaga, A. Alegría, A. J. Moreno, J. Colmenero, Macromolecules, 2011, 44, 8644; b) D. Chao, X. Jia, B. Tuten, C. Wang, E. B. Berda, Chem. Commun., 2013, 49, 4178 .

[28] Y. Oono, M. Kohmoto, J. Chem. Phys., 1983, 78, 520.

\section{Tables}


Table 1. Effect of precursor concentration on nanocarrier size during the synthesis of intrinsically disordered protein mimetics (IDPMs).

\begin{tabular}{|c|c|c|c|c|c|c|}
\hline System & $t_{R}(\mathbf{m i n})^{a}$ & $M_{w}(\mathbf{D a})^{b}$ & $M_{w} / M_{n}$ & $R_{g}(\mathbf{n m})^{\mathbf{c}}$ & $\begin{array}{c}R_{h} \\
(\mathbf{n m})^{\mathbf{d}}\end{array}$ & $\boldsymbol{R}_{g} / \boldsymbol{R}_{h}$ \\
\hline Precursor & 6.59 & $3.02 \times 10^{5}$ & 1.37 & 14.8 & 10.1 & 1.47 \\
\hline IDPM-3 $^{\mathrm{e}}$ & 6.76 & $3.22 \times 10^{5}$ & 1.33 & 10.6 & 8.5 & 1.25 \\
\hline IDPM-1 $^{\mathrm{f}}$ & 6.85 & $2.83 \times 10^{5}$ & 1.15 & 8.1 & 7.7 & 1.05 \\
\hline
\end{tabular}

${ }^{\text {a }}$ SEC retention time. ${ }^{\mathrm{b}}$ Absolute molecular weight at the SEC peak maximum. ${ }^{\mathrm{c}}$ Determined by SLS at the SEC peak maximum. ${ }^{\mathrm{d}}$ Determined by DLS. ${ }^{\mathrm{e}}$ Synthesis of IDPMs performed at $3 \mathrm{mg} / \mathrm{ml}$. ${ }^{\mathrm{f}}$ Synthesis of IDPMs performed at $1 \mathrm{mg} / \mathrm{ml}$.

\section{Figures}

A) 
<smiles>Nc1nc2ncc(CNc3ccc(C(=O)NC(CCC(=O)O)C(=O)O)cc3)nc2c(=O)[nH]1</smiles>

1<smiles>CC(C)c1cccc(=O)c(O)c1</smiles>

2

B)

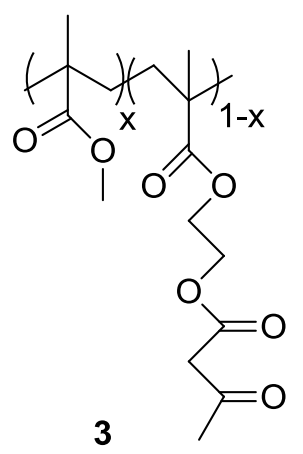

Fig. 1 A) Chemical structures of folic acid (1) and hinokitiol (2). B) Chemical structure of the single-chain nanocarrier precursor (3). 


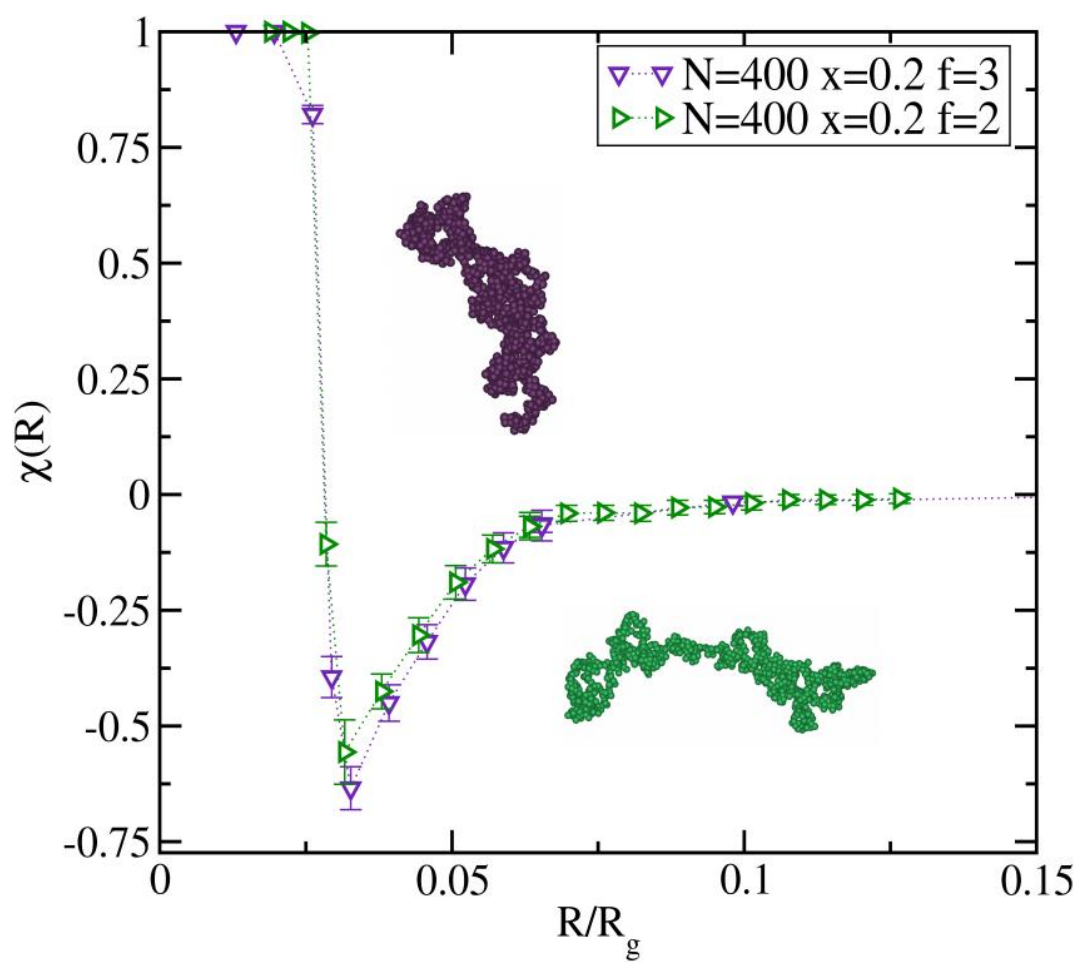

Fig. 2 Euler characteristic $\chi(\mathrm{R})$ of intrinsically disordered protein mimetics (IDPMs) as a function of $\mathrm{R} / \mathrm{R}_{\mathrm{g}}$. $\mathrm{R}$ is the appropriate length scale characterizing the size of the family of surfaces constructed from a given configuration of particles (see SI for details). IDPM configurations were determined from coarse-grained MD simulations under good solvent conditions for nanocarriers of $\mathrm{N}=400, \mathrm{x}=0.2, \mathrm{f}=2,\left\langle\mathrm{R}_{\mathrm{g}}{ }^{2}{ }^{1 / 2}=15.8 \sigma\right.$, and $\mathrm{N}=400, \mathrm{x}=0.2$, $\mathrm{f}=3,\left\langle\mathrm{R}_{\mathrm{g}}{ }^{2}\right\rangle^{1 / 2}=15.3 \sigma$. The snapshots represent typical conformations for $\mathrm{f}=3$ (top) and $\mathrm{f}=2$ (bottom). $(\mathrm{N}=$ number of beads, $\mathrm{x}=$ fraction of reactive groups in the chain, $\mathrm{f}=$ functionality, number of reactive groups that are allowed to be bonded per cross-linking point, $\mathrm{R}_{\mathrm{g}}=$ radius of gyration).

A) 


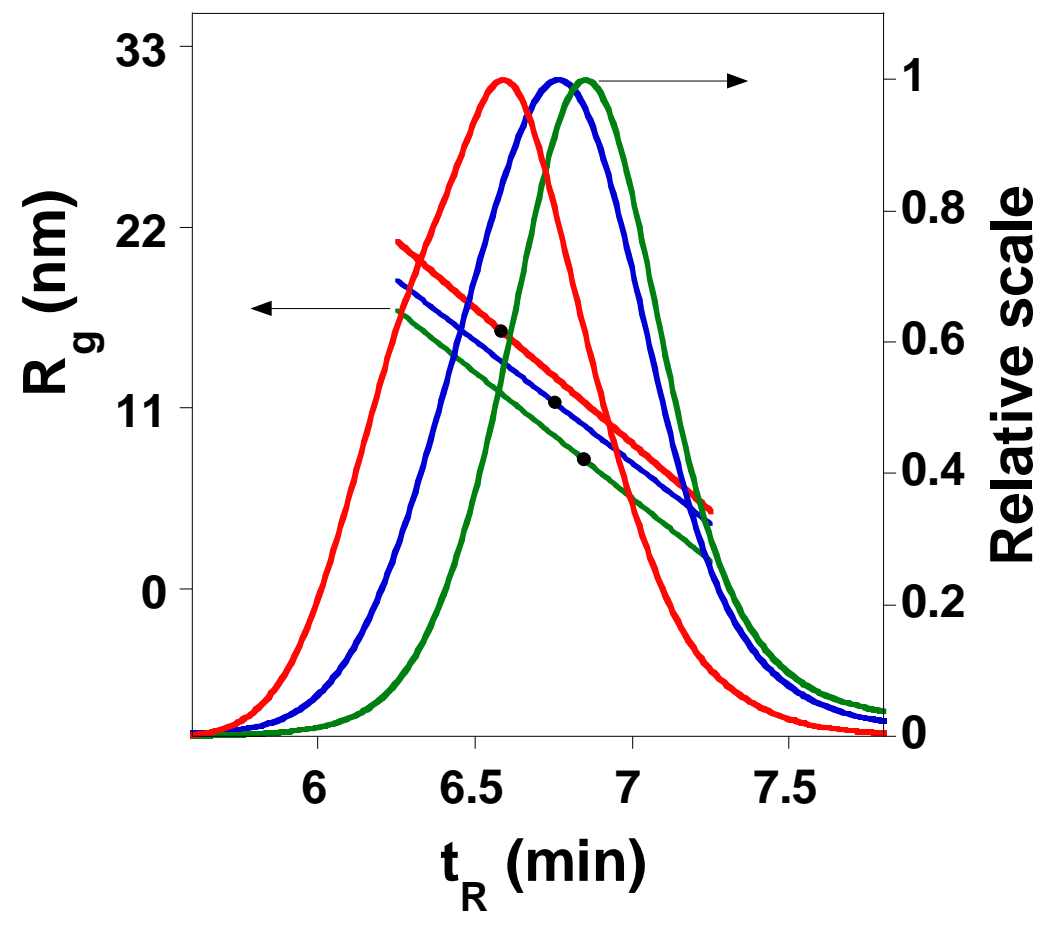

B)

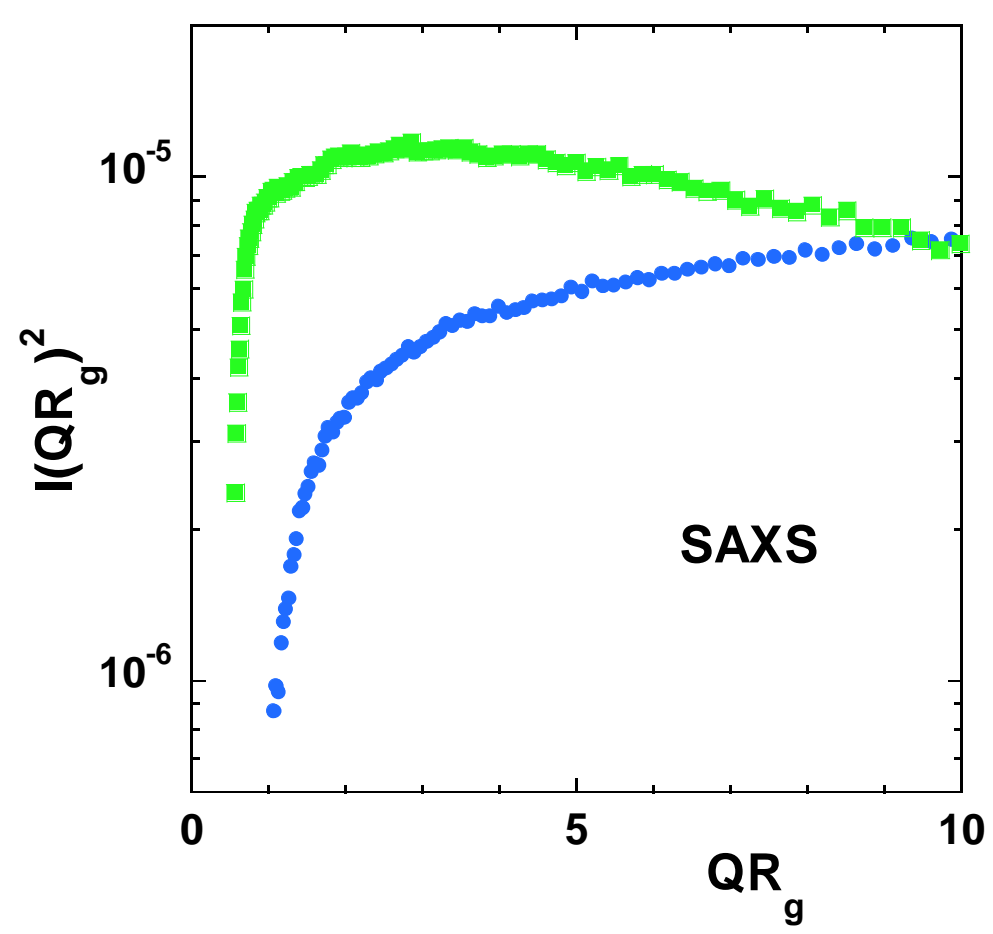

C) 


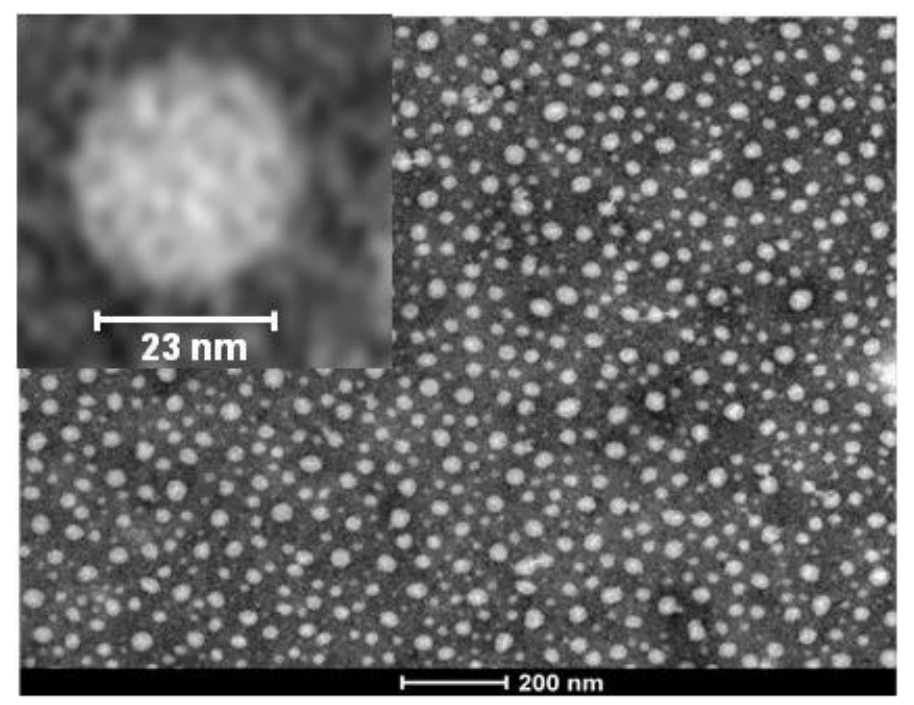

D)

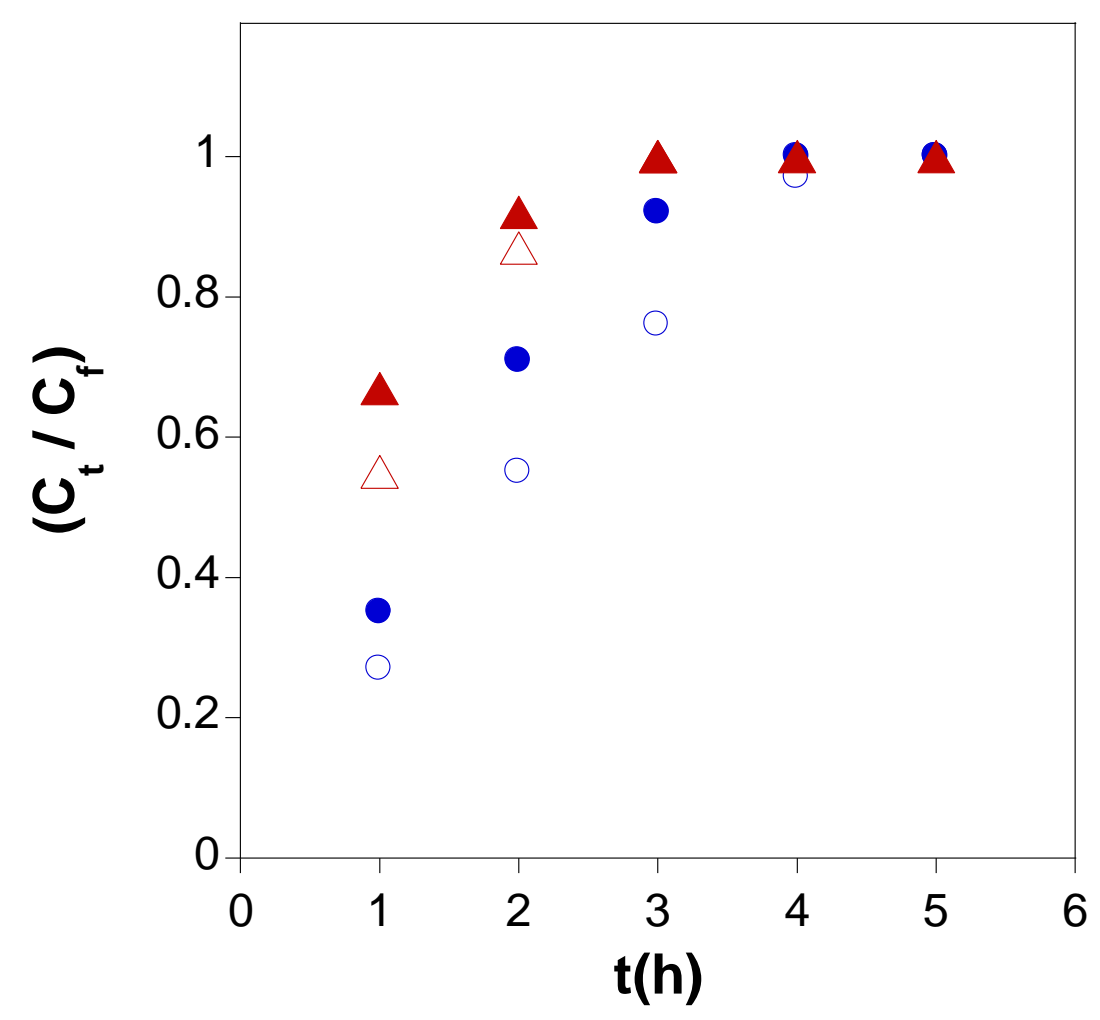

Fig. 3 Synthesis and characterization of intrinsically disordered protein mimetics (IDPMs) allowing simultaneous delivery of both dermal protective and anticancer cargos: A) 
Illustration of SEC/SLS data for the precursor (red lines), IDPMs synthesized at $3 \mathrm{mg} / \mathrm{ml}$ (blue lines) and IDPMs synthesized at $1 \mathrm{mg} / \mathrm{ml}$ (green lines). For each SEC trace, the value of $R_{g}$ at the peak maximum is indicated by a black circle. B) Kratky plot from small angle X-ray scattering (SAXS) measurements in THF of the precursor (blue line) and the corresponding IDPMs (green line). C) TEM picture showing the spherical shape of IDPMs onto a carboncoated grid upon solvent removal. D) Simultaneous delivery data in water at $\mathrm{pH}=6$ (blue symbols) and $\mathrm{pH}=8$ (red symbols) of folic acid, 1, (open symbols) and hinokitiol, 2, (solid symbols) from IDPMs. 
Simultaneous delivery of both dermal protective (folic acid) and anticancer (hinokitiol) cargos is reported from artificial intrinsically disordered protein mimics based on multidirectionally self-assembled single-chain nanocarriers.

Ana Sanchez-Sanchez, Somayeh Akbari, Angel J. Moreno, Federica Lo Verso, Arantxa Arbe, Juan Colmenero, José A. Pomposo*

Design and Preparation of Single-Chain Nanocarriers Mimicking Disordered Proteins for Combined Delivery of Dermal Bioactive Cargos

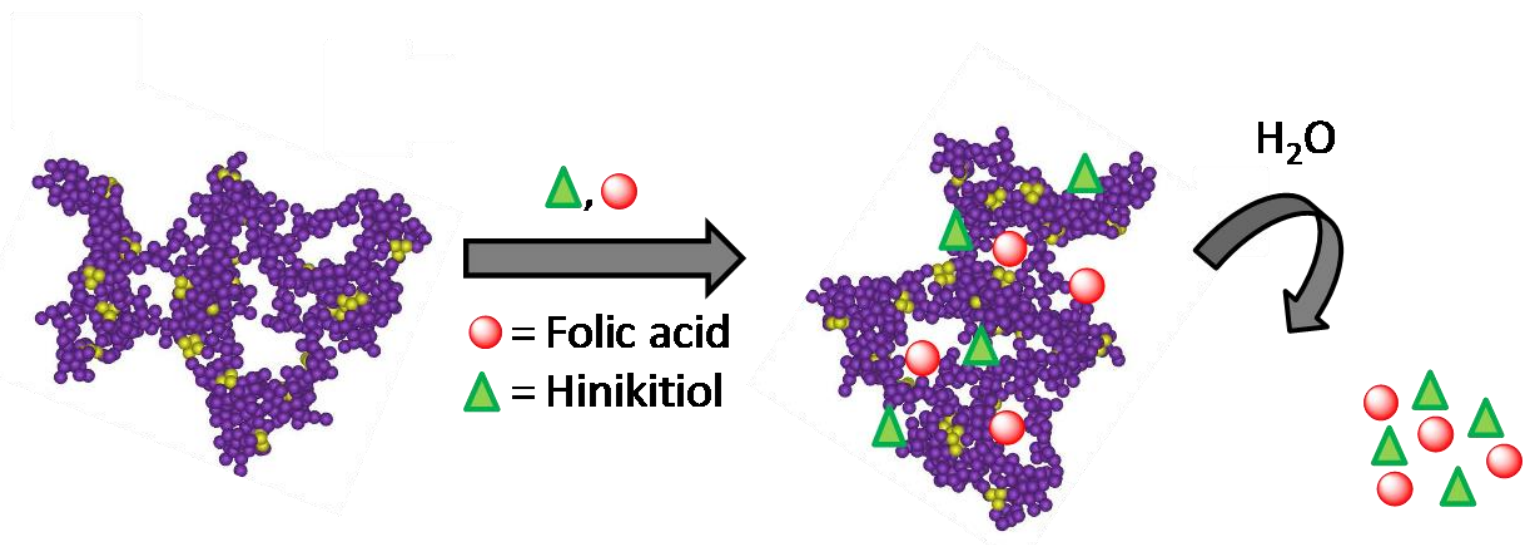

